\title{
Bone Tumor Pathology
}

Editor

JUDITH V.M.G. BOVÉE

\section{SURGICAL PATHOLOGY CLINICS}

www.surgpath.theclinics.com

Consulting Editor

JASON L. HORNICK

September 2017 • Volume 10 • Number 3 